\title{
PTH Signaling and Epigenetic Control of Bone Remodeling
}

\author{
Florante Ricarte $^{1} \cdot$ Teruyo Nakatani $^{2} \cdot$ Nicola Partridge ${ }^{1,2}$
}

Published online: 3 February 2016

(C) Springer International Publishing AG 2016

\begin{abstract}
As our understanding of the mechanisms that govern bone development advance, the role of epigenetic modifications in these processes becomes increasingly evident. Interestingly, in parathyroid hormone (PTH)-induced bone metabolism and remodeling, recent evidence shows that PTH signaling employs a particular facet of the epigenetic machinery to elicit its desired effects. In this review, we briefly discuss the known epigenetic events occurring in cells of the osteoblast lineage. More specifically, we elaborate on current findings that reveal the utilization of histone deacetylating enzymes (HDACs) in PTH-regulated modulation of gene expression in bone.
\end{abstract}

Keywords $\mathrm{PTH} \cdot \mathrm{HDAC} \cdot$ Transcriptional regulation $\cdot$ Bone remodeling

\section{Introduction}

In the past several years, studies in the field of epigenetics have illuminated a wide array of modifications that occur in the genome. More recently, the discovery of mechanisms such

This article is part of the Topical Collection on Molecular Biology of Skeletal Development

Nicola Partridge

ncp234@nyu.edu

1 Department of Biochemistry and Molecular Pharmacology, New York University School of Medicine, New York, NY 10016, USA

2 Department of Basic Science and Craniofacial Biology, New York University College of Dentistry, New York, NY 10010, USA as miRNA binding [1] and nucleosome positioning [2] have yielded surprising new ways in which the genome can be modified. However, the two most prominent modifications, DNA methylation and posttranslational modification of histones, have been extensively studied and thus, much information can be gleaned with respect to bone $[3,4 \bullet]$. Here, we focus on a specific subset of histone-modifying enzymes, histone deacetylases (HDACs), that work in corepressor complexes to modify chromatin structure and genomic availability and as discussed below, play a crucial role in PTH-regulated gene expression.

Parathyroid hormone (PTH) is an 84-amino-acid peptide hormone which acts as a vital regulator of calcium ion homeostasis and mediator of bone remodeling [5]. It is synthesized as a prepropeptide in the chief cells of the parathyroid glands and undergoes cleavage and transit through the endoplasmic reticulum and lastly, is stored in secretory vesicles $[6,7]$. The synthesis and release of PTH are governed by the calciumsensing receptor found on the plasma membrane of gland cells. This mechanism of calcium detection is highly sensitive, as minor changes in $\mathrm{Ca}^{2+}$ lead to large changes in PTH [8]. When serum $\mathrm{Ca}^{2+}$ levels are low, PTH is quickly exocytosed to restore a strict serum $\mathrm{Ca}^{2+}$ concentration of 1.1-1.3 mM. Additionally, serum phosphate, $1,25(\mathrm{OH})_{2}$ vitamin $\mathrm{D}_{3}$, and FGF23 are also able to modify PTH expression and secretion. Since phosphate is able to bind calcium, its presence in serum can result in a decrease in serum calcium, which promotes PTH secretion. Also, phosphate is able to stabilize PTH mRNA through a stabilizing complex (AUF1/UNR) that binds to the 3'-UTR of PTH [9]. $1,25(\mathrm{OH})_{2}$ vitamin $\mathrm{D}_{3}$ and its receptor, VDR, are able to bind regulatory elements in the PTH gene promoter and block transcription of PTH $[10,11]$. Lastly, FGF23 acts with coreceptors FGFR1c and $\alpha$-Klotho to directly reduce PTH mRNA levels, as well as PTH secretion [12]. 
PTH acts through its cognate receptor, PTHR1, a family B G protein-coupled receptor expressed primarily on osteoblasts and kidney cells [13]. PTHR1 mainly signals by coupling with the G $\alpha$ s/adenylyl cylase/cAMP/protein kinase A (PKA) pathway, but is also able to couple to G $\alpha \mathrm{q} /$ phospholipase C (PLC)/ inositol trisphosphate/cytoplasmic $\mathrm{Ca}^{2+}$ /protein kinase $\mathrm{C}$ (PKC) pathway [14]. The first 34 residues of PTH are able to recapitulate the biological activity of the full-length, mature polypeptide. Structurally, the C-terminus of PTH(1-34) interacts with the amino terminal, extracellular domain of PTHR1, while the N-terminus interacts with the transmembrane helices and extracellular loops [15•]. Similarly, parathyroid hormone-related protein (PTHrP) binds PTHR1 with the same affinity, but at different conformations of PTHR1 [16, 17]. It bears homology with PTH in the N-terminal 13 amino acids, which are involved in the activation of PTHR1, and due to this, PTHrP is being explored as an alternative to PTH in treating osteoporosis [18].

\section{PTH Signaling in Bone}

In order to utilize the calcium-rich bone matrix for maintenance of serum $\mathrm{Ca}^{2+}$ levels, the physiological actions of PTH on bone are largely catabolic. Through its actions on osteoblasts and osteocytes, PTH is able to indirectly activate osteoclasts and promote bone resorption. One of the major pathways utilized in PTH-induced resorption is the receptor activator of nuclear factor-kappa B ligand (RANKL)/Rank/ osteoprotegerin (OPG) system. RANKL binds its cognate receptor, RANK, which is found on the osteoclast surface, to induce osteoclastogenesis and osteoclast activation, subsequently resulting in bone resorption $[19,20]$. To counter these effects, osteoblasts are able to express OPG, a soluble decoy receptor, to inhibit RANKL binding to RANK on osteoclasts and in doing so, decreases bone resorption [21]. PTH has been found to modulate this ratio in differentiating osteoblasts by suppressing OPG expression in early osteoblasts and increasing RANKL expression in mature osteoblasts, which ultimately results in an increase in bone resorption [22]. Additionally, previous reports revealed that the expression and enzymatic activity of matrix metalloproteinase-13 (MMP-13), an enzyme responsible for the degradation of bone extracellular matrix, are induced by PTH $[23,24]$. MMP-13 is a complex secondary response gene, and its activation is dependent upon Runx2 and Jun/Fos, as well as several phosphorylation events executed by PKA. Prior work in our laboratory has found that the PTH regulatory region lies 148-bp upstream of the transcriptional start site [25], but more recent studies reveal enhancer regions $-10,-20$, and even $-30 \mathrm{~kb}$ upstream of the Mmp13 promoter and are utilized by $\operatorname{VDR}, \mathrm{C} / \mathrm{EBP} \beta$, and Runx2, respectively [26].

Conversely, PTH is also able to exert anabolic effects on bone. This was revealed through the study of sclerostin, a glycoprotein secreted mainly by osteocytes. Encoded by the SOST gene, the main function of sclerostin is to inhibit bone formation through inhibition of canonical $\mathrm{Wnt} / \beta$-catenin osteoanabolic signaling [27-31]. As such, functional mutation of SOST in humans leads to two autosomal recessive disorders, sclerosteosis and Van Buchem disease, both of which are characterized by severe bone overgrowth [32, 33]. Remarkably, PTH was found to inhibit Sost and this action was essential for the osteoanabolic effects of PTH [34-36].

Notably, the periodicity and length of administration of PTH renders the hormone catabolic or anabolic. The continuous administration of PTH leads to an increase in bone resorption and catabolic effects [37]. However, and in contrast to its physiological role, PTH is able to exert anabolic effects on the skeleton upon intermittent administration and is currently the only osteoanabolic therapy available for the treatment of osteoporosis [38]. Nonetheless, PTH achieves either effect through the regulation of key anabolic or catabolic/ osteoclastogenic genes. As such, recent evidence suggests that some of these regulatory pathways elicit the use of histone deacetylating enzymes, or HDACs.

\section{HDACs in Bone Development and Remodeling}

HDACs belong to a superfamily of enzymes that play vital roles in several cellular processes, mainly through their repressive effect on transcription $[39,40]$. They act in converse with a family of enzymes, the histone acetyltransferases (HATs), which enhance transcription by catalyzing the transfer of acetyl groups from acetyl coenzyme A (Acetyl CoA) to epsilonamino groups of lysine residues within histone tails. This neutralizes their inherently positive charge, which in turn relaxes chromatin structure. In disrupting the structural integrity of the chromatin, transcription factors are free to bind their target sequences [41]. In addition, acetylated histones may serve as binding sites for bromodomain proteins, which can serve as transcriptional activators. Thus, removal of acetyl groups by HDACs leads to chromatin compaction and blocks any factors that promote transcription. The intricate balance between HATs and HDACs is a key mechanism in governing gene expression.

The mammalian HDAC superfamily consists of 18 proteins and is classified into the following subfamilies: class I includes HDACs 1-3, 8; class IIa includes HDACs 4, 5, 7, 9; class IIb includes 6, 10; class IV includes HDAC11; and lastly, class III includes sirtuins 1-7 [42, 43]. The HDACs differ in expression patterns, enzymatic functions, structure, and subcellular location. HDAC classes I, II, and IV require $\mathrm{Zn}^{2+}$, while the sirtuins require the cofactor nicotinamide adenine dinucleotide (NAD+) and are intriguingly regarded as a family of genes involved in aging [see 44, 45 for reviews]. 
Interestingly, HDAC activity is not limited to histones. Indeed, they are able to target lysine residues found on nonhistone proteins. Because HDACs lack the ability to directly bind chromatin, they rely on proteins that do bind DNA to form complexes to control gene expression, particularly in osteoblast differentiation [46-48]. Specifically, one of the targets in bone is a master transcription factor, Runx2. This action is mediated through deacetylation and/or direct binding of HDACs to Runx2 [49-51]. Runx2 belongs to the Runt related family of transcription factors (RUNX), and these are characterized by a 128 -amino-acid domain (runt domain), which binds an RD consensus sequence, PuACCPuCA [52]. The RUNX family is involved in a wide array of functions and systems, but Runx 2 has been characterized as an osteoblastspecific transcriptional activator $[53,54]$ and plays a central role in osteoblast differentiation $[55,56]$.

Classes I, IIa, and IIb HDACs are expressed in osteoblasts and bind Runx2 [49, 57, 58], and these interactions appear to be of great importance during osteoblast maturation. In an effort to identify corepressors that bind Runx2, Schroeder et al. found that HDAC 3 was able to bind the N-terminus of Runx2, but did not associate with the Runt domain itself [49]. Indeed, HDAC3 blocks the activation of the osteocalcin promoter by binding to a region with a Runx 2 binding site within it and this repression was blocked with either HDAC inhibitors or shRNAs targeting HDAC3 specifically [49].

By interacting with a Runx $2 / \mathrm{Smad} 3$ complex, HDAC4 and HDAC5 appear to regulate Tgf- $\beta$-dependent signaling and gene expression [59]. Previously, Westendorf et al. showed that in differentiated osteoblasts, HDAC6 localizes to the nucleus and binds Runx2 in a rapid, but transient manner [57]. Also, deficiencies in HDAC6 lead to an increase in trabecular bone density [60]. By stimulating the phosphorylation of HDAC7 by protein kinase $\mathrm{D}, \mathrm{Bmp} 2$ disrupts the interaction between HDAC7 and Runx2, prompting the association of HDAC7 with 14-3-3 proteins, leading to its exit from the nucleus [61]. This same study also reported that Bmp2 leads to the nuclear exit of HDAC4, but did not affect the subcellular localization of HDAC5 or HDAC6. Studies in our laboratory have shown that HDAC4 controls the activity of matrix metalloproteinase-13 (MMP13) through dissociation from Runx2, and this regulation is governed by $\mathrm{PTH}$ in a protein kinase A (PKA) and protein phosphatase 2A (PP2A)-dependent manner $[62,63 \bullet]$. Thus, these studies corroborate a defining characteristic of class II HDACs, in which their regulation is governed by subcellular localization.

Of all the class III HDACs, SIRT1 has been the most heavily investigated. Indeed, it appears to play a significant role in bone development, as global deletion of SIRT1 in mice leads to delayed bone mineralization [64]. Additionally, knockout of Sirt1 in osteoblast-specific or mesenchymal stem cellspecific mice results in a significant loss of bone mass [65, 66]. Recent studies suggest that SIRT1 action is also mediated through Runx2 $[67,68]$. Taken together, the prominent role of HDACs in bone remodeling suggests their likely utilization in PTH-mediated actions on bone.

\section{HDACs in PTH-Mediated Bone Remodeling}

It is well understood that PTH is able to produce anabolic and catabolic effects on bone, depending on the duration and periodicity of its administration. Continuous treatment creates a catabolic effect, where high bone turnover is stimulated, whereas intermittent treatments increase bone formation [37]. Thus, daily intermittent injections of $\mathrm{PTH}(1-34)$ remain the only FDA-approved osteoanabolic therapy [38]. Although the effect of intermittent treatment of $\mathrm{PTH}(1-34)$ is well known, the precise mechanisms by which bone formation prevail are poorly understood. Remarkably, studies in PTH signaling in the osteoblast lineage have been shown to regulate class IIa HDACs, HDAC4 and HDAC5, and class III HDAC, Sirtuin 1, and these findings may, in part, aid in furthering understanding the effects of PTH on bone.

As discussed above, studies in our laboratory have shown that PTH, by way of PKA-dependent phosphorylation, utilizes HDAC4 to control Mmp13 expression in the rat osteoblastic cell line, UMR 106-01. Phosphorylation of HDAC4 on Ser632 (Ser-740 in rats) is a critical event that causes the release of HDAC4 from Runx 2 on the Mmp13 promoter. In addition, dephosphorylation of HDAC4 on Ser-246 (Ser-355 in rats) leads to its subsequent translocation out of the nucleus [63]. Together, these events allow for the recruitment of histone acetyltransferases (HATs) such as $\mathrm{p} 300$ and P/CAF to promote transcription $[69,70]$. Conversely, Obri et al. reported that in mouse calvarial cells, HDAC4 does not inhibit Runx2 function but rather promotes RankL expression through PTH-induced, Smurf2-mediated polyubiquitylation of HDAC4 and its subsequent degradation. Alternatively, the sympathetic tone promotes HDAC4 nuclear accumulation and association with transcription factor, ATF4 [71]. Interestingly, a recent study found that proteasome inhibitor carfilzomib (CFZ) was able to inhibit PTH-induced RANKL expression by preventing HDAC4 degradation in the osteoblast [72], thus inhibiting osteoclastogenesis and suggests the possible combinatorial use of CFZ and PTH in therapy.

Recently, a large transcriptome study was performed to assess the comparative genetic expression elicited by either PTH or $1,25(\mathrm{OH})_{2}$ vitamin $\mathrm{D}_{3}$. This study concluded that PTH and $1,25(\mathrm{OH})_{2}$ vitamin $\mathrm{D}_{3}$ collaborate to simultaneously limit osteocyte differentiation of precursor cells and promote mature osteocyte function [73•]. Additionally, they also report the identification of a PKA-activated phospho-Creb (pCREB) cistrome, which revealed that despite many differentiationrelated PTH regulated genes were dependent on the PKAmediated signaling pathway, a number of pCREB binding 
sites associated with osteocyte-specific PTH targets appeared to involve alternative pathways.

PTH signaling also appears to employ the use of another class IIa HDAC, HDAC5. Baertschi et al. report that PTH induces nuclear translocation of HDAC5, colocalization with MEF2s, and subsequent sclerostin suppression. Conversely, inhibition of class I HDACs by siRNA, HDAC2 and HDAC3, led to a decrease in Sost suppression, which implicates their involvement in Sost expression [74•]. It is important to note that the cells used in this study, UMR 106-01, were described as osteocytic in nature, when in fact, they are osteoblastic cells derived from a rat osteosarcoma. Supporting some of this group's work, a more recent study showed that HDAC5 knockout mice exhibit increased levels of SOST mRNA, as well as reduced bone formation by osteoblasts. Additionally, chromatin immunoprecipitation studies performed in an osteocytic cell line, Ocy454, reveal an inhibitory relationship between $\mathrm{HDAC} 5$ and the binding of MEF2C to a distal intergenic enhancer region within SOST [75•].

A recent study in our laboratory has implicated SIRT1 in the PTH-regulated expression of MMP13 [76•]. Sirt1 knockout mice exhibited an increase in both MMP13 mRNA expression, as well as protein levels. By using a SIRT1 activator, resveratrol, or SIRT1 inhibitor, EX527, we were able to either block or enhance PTH-stimulated MMP13 expression, respectively. After treating osteoblastic cells with $\mathrm{PTH}$, we observed binding of SIRT1 with c-Jun, which is a component of the transcription factor complex, activator protein 1 (AP-1). This association at the AP-1 site of the Mmp13 promoter inhibits AP-1 activity and thus, downregulates the transcription of Mmp13. Also, inhibition of SIRT1 led to an increase in PTH-stimulated Mmp13 gene expression. Interestingly, we also found that SIRT1 deacetylates c-Jun in a cAMPdependent manner. These data conclude that SIRT1 is a negative regulator of MMP13 expression, and this regulation is achieved through the direct binding of SIRT1 with c-Jun on the Mmp13 promoter.

\section{Conclusions}

Recent studies have shown that the role of HDACs in PTHinduced gene regulation is abundantly clear, particularly with class II HDACs. It is interesting to note that of all the HDACs, these exhibit relatively low expression and are the most spatiotemporally specific. Also, this subfamily possesses the least enzymatic activity and appears to be used more for their ability to bind transcription factors and block recruitment of transcriptional activators. Studies on their ability to occupy promoter sites and the effect this has on blocking histone acetyltransferase access would be insightful. With respect to class III HDACs, Sirt1 plays a significant role in inhibiting a catabolic action of PTH on bone, which may be a potential target in the treatment of osteoporosis. Additional studies on the role of HDACs in PTH signaling would offer a wealth of knowledge, as further understanding of these precise mechanisms would provide novel therapeutic targets in bone disease and treatment.

Acknowledgments This work was funded in part by NIH grant DK47420 to NCP.

\section{Compliance with Ethical Standards}

Conflict of Interest Florante Ricarte and Teruyo Nakatani declare that they have no conflict of interest.Nicola Partridge reports grants from NIH, during the conduct of the study.

Human and Animal Rights and Informed Consent This article does not contain studies with human subjects performed by the authors. The article does contain studies with animals for which two of the authors were authors. These studies were performed following protocols approved by NYU Institutional Animal Care and Use Committee (IACUC).

\section{References}

Papers of particular interest, published recently, have been highlighted as:

- Of importance

1. Miao CG, Yang YY, He X, Xu T, Huang C, Huang Y, et al. New advances of microRNAs in the pathogenesis of rheumatoid arthritis, with a focus on the crosstalk between DNA methylation and the microRNA machinery. Cell Signal. 2013;25:1118-25.

2. Struhl K, Segal E. Determinants of nucleosome positioning. Nat Struct Mol Biol. 2013;20:267-73.

3. Rothbart SB, Strahl BD. Interpreting the language of histone and DNA modifications. Biochim Biophys Acta. 1839;2014:627-43.

4. Gordon F, Stein J, Westendorf J, van Wijnen A. Chromatin modifiers and histone modifications in bone formation, regeneration, and therapeutic intervention for bone related disease. Bone. 2015. doi: 10.1016/j.bone.2015.03.011. An excellent review on the entire HDAC family in bone development and remodeling.

5. Strewler GJ, Stern PH, Jacobs JW, Eveloff J, Klein RF, Leung SC, et al. Parathyroid hormone like protein from human renal carcinoma cells. Structural and functional homology with parathyroid hormone. J Clin Invest. 1987;80:1803-7.

6. Habener JF, Potts Jr JT, Rich A. Pre-proparathyroid hormone. Evidence for an early biosynthetic precursor of pro parathyroid hormone. J Biol Chem. 1976;251:3893-9.

7. Kemper B, Habener JF, Rich A, Potts Jr JT. Microtubules and the intracellular conversion of pro parathyroid hormone to parathyroid hormone. Endocrinology. 1975;96:903-12.

8. Diaz R, El-Hajj GF, Brown E. Regulation of parathyroid hormone function. In: Fray JGS, editor. Handbook of physiology. New York: Oxford University Press; 1998. p. 607-62.

9. Navh-Many T. Minireview: the play of proteins on the parathyroid hormone messenger ribonucleic acid regulates its expression. Endocrinology. 2010;151:1398-402.

10. Kim MS, Fujiki R, Murayama A, Kitagawa H, Yamaoka K, Yamamoto $\mathrm{Y}$, et al. 1Alpha,25(OH)2D3-induced transrepression by vitamin $\mathrm{D}$ receptor through E-box-type elements in the human 
parathyroid hormone gene promoter. Mol Endocrinol. 2007;21: 334-42.

11. Okazaki T, Igarashi T, Kronenberg HM. 5'-flanking region of the parathyroid hormone gene mediates negative regulation by $1,25-$ (OH)2 vitamin D3. J Biol Chem. 1988;263:2203-8.

12. Ben-Dov IZ, Galitzer H, Lavi-Moshayoff V, Goetz R, Kuro-o M, Mohammadi M, et al. The parathyroid is a target organ for FGF23 in rats. J Clin Invest. 2007;117:4003-8.

13. Jüppner H. Abou-Samra AB, Freeman M, Kong XF, Schipani E, Richards J, Kolakowski LF Jr, Hock J, Potts JT, Jr, Kronenberg HM, Segre GV (1991) A G protein-linked receptor for parathyroid hormone and parathyroid hormone-related peptide. Science. 1991;254:1024-6.

14. Bisello A, Chorev M, Rosenblatt M, Monticelli L, Mierke DF, Ferrari SL. Selective ligand-induced stabilization of active and desensitized parathyroid hormone type I receptor conformations. J Biol Chem. 2002;277:38524-30.

15. Gardella T, Vilardaga JP. International union of basic and clinical pharmacology. XCIII. The parathyroid hormone receptors- family B G protein-coupled receptors. Pharmacol Rev. 2015;67:310-37. An extensive review on PTHR1 structure, as well as PTH and PTHrP binding.

16. Okazaki M, Ferrandon S, Vilardaga JP, Bouxsein M, Potts Jr J, Gardella T. Prolonged signaling at the parathyroid hormone receptor by peptide ligands targeted to a specific receptor conformation. Proc Natl Acad Sci U S A. 2008;105(43):16525-30.

17. Dean T, Vilardaga JP, Potts Jr J, Gardella T. Altered selectivity of parathyroid hormone (PTH) and $\mathrm{PTH}$-related protein (PTHrP) for distinct conformations of the $\mathrm{PTH} / \mathrm{PTHrP}$ receptor. Mol Endocrinol. 2008;22(1):156-66.

18. Esbrit P, Herrera S, Portal-Nunez S, Nogues X, Diez-Perez A. Parathyroid hormone-related protein analogs as osteoporosis therapies. Calcif Tissue Int. 2015. doi:10.1007/s00223-015-0050-1.

19. Nakagawa N, Kinosaki M, Yamaguchi K, Shima N, Yasuda H, Yano K, et al. RANK is the essential signaling receptor for osteoclast differentiation factor in osteoclastogenesis. Biochem Biophys Res Commun. 1998;253:395-400.

20. Hsu H, Lacey DL, Dunstan CR, Solovyev I, Colombero A, Timms E, et al. Tumor necrosis factor receptor family member RANK mediates osteoclast differentiation and activation induced by osteoprotegerin ligand. Proc Natl Acad Sci U S A. 1999;96:3540-5.

21. Simonet WS, Lacey DL, Dunstan CR, Kelley M, Chang M-S, Luthy R, et al. Osteoprotegerin: A novel secreted protein involved in the regulation of bone density. Cell. 1997;89:309-19.

22. Huang J, Sakata T, Pfleger L, Bencsik M, Halloran B, Bikle D, et al. PTH differentially regulated expression of RANKL and OPG. J Bone Miner Res. 2004;19:235-44.

23. Partridge NC, Jeffrey JJ, Ehlich LS, Teitelbaum SL, Fliszar C, Welgus HG, et al. Hormonal regulation of the production of collagenase and a collagenase inhibitor activity by rat osteogenic sarcoma cells. Endocrinology. 1987;120:1956-62.

24. Scott DK, Brakenhoff KD, Clohisy JC, Quinn CO, Partridge NC. Parathyroid hormone induces transcription of collagenase in rat osteoblastic cells by a mechanism using cyclic adenosine 3',5'monophosphate and requiring protein synthesis. Mol Endocrinol. 1992;6(12):2153-9.

25. Selvamurugan N, Chou WY, Pearman AT, Pulumati MR, Partridge NC. Parathyroid hormone regulates the rat collagenase-3 promoter in osteoblastic cells through the cooperative interaction of the activator protein-1 site and the runt domain binding sequence. J Biol Chem. 1998;273:10647-57.

26. Meyer M, Benkusky NA, Pike JW. Selective distal enhancer control of the Mmp13 gene identified through clustered regularly interspaced short palindromic repeat (CRISPR) genomic deletions. J Biol Chem. 2015;290(17):11093-107.
27. Kramer I, Halleux C, Keller H, Pegurri M, Gooi JH, Weber PB, et al. Osteocyte Wnt/beta-catenin signaling is required for normal bone homeostasis. Mol Cell Biol. 2010;30:3071-85.

28. Glass 2nd DA, Bialek P, Ahn JD, Starbuck M, Patel MS, Clevers H, et al. Canonical Wnt signaling in differentiated osteoblasts controls osteoclast differentiation. Dev Cell. 2005;8:751-64

29. Holmen SL, Zylstra CR, Mukherjee A, Sigler RE, Faugere MC, Bouxsein ML, et al. Essential role of beta-catenin in postnatal bone acquisition. J Biol Chem. 2005;280:21162-8.

30. Poole KE, van Bezooijen RL, Loveridge N, Hamersma H, Papapoulos SE, Lowik CW, et al. Sclerostin is a delayed secreted product of osteocytes that inhibits bone formation. FASEB J. 2005;19:1842-4.

31. Costa AG, Bilezikian JP. Sclerostin: therapeutic horizons based upon its actions. Curr Osteoporos Rep. 2012;10:64-72.

32. Balemans W, Ebeling M, Patel N, Van Hul E, Olson P, Dioszegi M, et al. Increased bone density in sclerosteosis is due to the deficiency of a novel secreted protein (SOST). Hum Mol Genet. 2001;10:53743.

33. Balemans W, Patel N, Ebeling M, Van Hul E, Wuyts W, Lacza C, et al. Identification of a $52 \mathrm{~kb}$ deletion downstream of the SOST gene in patients with van Buchem disease. J Med Genet. 2002;39:91-7.

34. Bellido T, Ali AA, Gubrij I, Plotkin LI, Fu Q, O'Brien CA, et al. Chronic elevation of parathyroid hormone in mice reduces expression of sclerostin by osteocytes: a novel mechanism for hormonal control of osteoblastogenesis. Endocrinology. 2005;146:4577-83.

35. Powell Jr WF, Barry KJ, Tulum I, Kobayashi T, Harris SE, Bringhurst FR, et al. Targeted ablation of the PTH/PTHrP receptor in osteocytes impairs bone structure and homeostatic calcemic responses. J Endocrinol. 2011;209:21-32.

36. Keller $\mathrm{H}, \mathrm{Kneissel} \mathrm{M.} \mathrm{SOST} \mathrm{is} \mathrm{a} \mathrm{target} \mathrm{gene} \mathrm{for} \mathrm{PTH}$ in bone. Bone. 2005;37:148-58.

37. Silva BC, Bilezikian JP. Parathyroid hormone: anabolic and catabolic actions on the skeleton. Curr Opin Pharmacol. 2015;22:4150 .

38. Neer RM, Arnaud CD, Zanchetta JR, Prince R, Gaich GA, Reginster JY, et al. Effect of parathyroid hormone (1-34) on fractures and bone mineral density in postmenopausal women with osteoporosis. N Engl J Med. 2001;344:1434-41.

39. Haberland M, Montgomery R, Olson E. The many roles of histone deacetylases in development and physiology: implications for disease and therapy. Nat Rev Genet. 2009;10(1):32-42.

40. Bradley EW, McGee-Lawrence ME, Westendorf JJ. Hdac-mediated control of endochondral and intramembranous ossification. Crit Rev Eukaryot Gene Expr. 2011;21:101-13.

41. Shahbazian MD, Grunstein M. Functions of site-specific histone acetylation and deacetylation. Annu Rev Biochem. 2007;76:75100.

42. Gray SG, Ekstrom TJ. The human histone deacetylase family. Exp Cell Res. 2001;262:75-83.

43. Gregoretti IV, Lee YM, Goodson HV. Molecular evolution of the histone deacetylase family: Functional implications of phylogenetic analysis. J Mol Biol. 2004;338:17-31.

44. Schwer B, Verdin E. Conserved metabolic regulatory functions of sirtuins. Cell Metab. 2008;7:104-12.

45. Longo VD, Kennedy BK. Sirtuins in aging and age-related disease. Cell. 2006;126:257-68.

46. Choo MK, Yeo H, Zayzafoon M. NFATc1 mediates HDACdependent transcriptional repression of osteocalcin expression during osteoblast differentiation. Bone. 2009;45:579-89.

47. Hesse E, Saito H, Kiviranta R, Correa D, Yamana K, Neff L, et al. Zfp521 controls bone mass by HDAC3-dependent attenuation of Runx2 activity. J Cell Biol. 2010;191:1271-83.

48. Jensen ED, Schroeder TM, Bailey J, Gopalakrishnan R, Westendorf JJ. Histone deacetylase 7 associates with Runx2 and represses its 
activity during osteoblast maturation in a deacetylationindependent manner. J Bone Miner Res. 2008;23:361-72.

49. Schroeder TM, Kahler RA, Li X, Westendorf JJ. Histone deacetylase 3 interacts with runx 2 to repress the osteocalcin promoter and regulate osteoblast differentiation. J Biol Chem. 2004;279:41998-2007.

50. Fu Y, Zhang P, Ge J, Cheng J, Dong W, Yuan H, et al. Histone deacetylase 8 suppresses osteogenic differentiation of bone marrow stromal cells by inhibiting histone H3K9 acetylation and RUNX2 activity. Int J Biochem Cell Biol. 2014;54:68-77.

51. Bradley EW, Carpio LR, Olson EN, Westendorf JJ. Histone deacetylase 7 (Hdac7) suppresses chondrocyte proliferation and $\beta$-catenin activity during endochondral ossification. J Biol Chem. 2015;290:118-26.

52. Kagoshima H, Satake M, Miyoshi H, Ohki M, Pepling M, Gergen $\mathrm{JP}$, et al. The runt domain identifies a new family of heteromeric transcriptional regulators. Trends Genet. 1993;9:338-41.

53. Satake M, Nomura S, Yamaguchi-Iwai Y, Takahama Y, Hashimoto Y, Niki M, et al. Expression of the runt domain-encoding PEBP2a genes in $\mathrm{T}$ cells during thymic development. Mol Cell Biol. 1995; 15:1662-70.

54. Ducy P, Zhang R, Geoffroy V, Ridall AL, Karsenty G. Osf2/ Cbfa1: A transcriptional activator of osteoblast differentiation. Cell. 1997;89:747-54

55. Komori T, Yagi H, Nomura S, Yamaguchi A, Sasaki K, Deguchi K, et al. Targeted disruption of Cbfal results in a complete lack of bone formation owing to maturational arrest of osteoblasts. Cell. 1997;89(5):755-64.

56. Otto F, Thornell AP, Crompton T, Denzel A, Gilmour KC, Rosewell IR, et al. Cbfa1, a candidate gene for cleidocranial dysplasia syndrome, is essential for osteoblast differentiation and bone development. Cell. 1997;89(5):765-71.

57. Westendorf JJ, Zaidi SK, Cascino JE, Kahler R, van Wijnen AJ, Lian JB, et al. Runx2 (Cbfa1, AML-3) interacts with histone deacetylase 6 and represses the p21(CIP1/WAF1) promoter. Mol Cell Biol. 2002;22(22):7982-92.

58. Westendorf JJ. Transcriptional co-repressors of Runx2. J Cell Biol. 2006;98(1):54-64.

59. Kang JS, Alliston T, Delston R, Derynck R. Repression of Runx2 function by TGF-beta through recruitment of class II histone deacetylases by Smad3. EMBO J. 2005;24(14):2543-55.

60. Zhang Y, Kwon S, Yamaguchi T, Cubizolles F, Rousseaux S, Kneissel M, et al. Mice lacking histone deacetylase 6 have hyperacetylated tubulin but are viable and develop normally. Mol Cell Biol. 2008;28(5):1688-701.

61. Jensen ED, Gopalakrishnan R, Westendorf JJ. Bone morphogenic protein 2 activates protein kinase $\mathrm{D}$ to regulate histone deacetylase 7 localization and repression of Runx2. J Biol Chem. 2009;284(4): 2225-34.

62. Shimizu E, Selvamurugan N, Westendorf JJ, Olson EN, Partridge NC. HDAC4 represses matrix metalloproteinase-13 transcription in osteoblastic cells, and parathyroid hormone controls this repression. J Biol Chem. 2010;285(13):9616-26.

63. Shimizu E, Nakatani T, He Z, Partridge NC. Parathyroid hormone regulates histone deacetylase (HDAC) 4 through protein kinase Amediated phosphorylation and dephosphorylation in osteoblastic cells. J Biol Chem. 2014;289(31):21340-50. Describes PKAdependent PTH actions on HDAC4.

64. Lemieux ME, Yang X, Jardine K, He X, Jacobsen KX, Staines WA, et al. The Sirt1 deacetylase modulates the insulin-like growth factor signaling pathway in mammals. Mech Ageing Dev. 2005;126: 1097-105.

65. Edwards JR, Perrien DS, Fleming N, Nyman JS, Ono K, Connelly $\mathrm{L}$, et al. Silent information regulator (Sir)T1 inhibits NFKB signaling to maintain normal skeletal remodeling. J Bone Miner Res. 2013;28:960-9.

66. Simic P, Zainabadi K, Bell E, Sykes DB, Saez B, Lotinun S, et al. SIRT1 regulates differentiation of mesenchymal stem cells by deacetylating B-catenin. EMBO Mol Med. 2013;5:430-40.

67. Shakibaei M, Shayan P, Busch F, Aldinger C, Buhrmann C, Lueders C, et al. Resveratrol mediated modulation of Sirt-1/ Runx2 promotes osteogenic differentiation of mesenchymal stem cells: potential role of Runx2 deacetylation. PLoS One. 2012;7: e35712.

68. Tseng PC, Hou SM, Chen RJ, Peng HW, Hsieh CF, Kuo ML, et al. Resveratrol promotes osteogenesis of human mesenchymal stem cells by upregulating RUNX2 gene expression via the SIRT1/ FOXO3A axis. J Bone Miner Res. 2011;26:2552-63.

69. Boumah CE, Lee M, Selvamurugan N, Shimizu E, Partridge NC. Runx2 recruits $\mathrm{p} 300$ to mediate parathyroid hormone's effects on histone acetylation and transcriptional activation of the matrix metalloproteinase-13 gene. Mol Endocrinol. 2009;23:1255-63

70. Lee N, Partridge NC. Parathyroid hormone activation of matrix metalloproteinase- 13 requires the histone acetyltransferase activity of $\mathrm{p} 300$ and PCAF and p300 dependent acetylation of PCAF. J Biol Chem. 2010;285:38014-22.

71. Obri A, Makinistoglu M, Zhang H, Karsenty G. HDAC4 integrates PTH and sympathetic signaling in osteoblasts. J Cell Biol. 2014;205(6):771-80.

72. Yang Y, Blair H, Shapiro I, Wang B. The proteasome inhibitor carfilzomib suppresses parathyroid hormone-induced osteoclastogenesis through a RANKL-mediated signaling pathway. J Biol Chem. 2015;290(27):16918-28.

73. St. John H, Meyer M, Benkusky N, Carlson A, Prideaux M, Bonewald L, et al. The parathyroid hormone-regulated transcriptome in osteocytes: parallel actions with 1,25 dihydroxyvitamin D3 to oppose gene expression changes during differentiation and to promote mature cell function. Bone. 2015;72:81-91. A thorough study examining the collaborative effects of PTH and 1,25-dihydroxyvitamin D3.

74. Baertschi S, Baur N, Lueders-Lefevre V, Voshol J, Keller H. Class I and Ila histone deacetylases have opposite effects on sclerostin gene regulation. J Biol Chem. 2014;289(36):24995-5009. Implicates HDAC5 in PTH-mediated regulation of SOST.

75. Wein M, Spatz J, Nishimori S, Doench J, Root D, Babij P, et al. HDAC5 controls MEF2C-driven sclerostin expression in osteocytes. J Bone Miner Res. 2015;30(3):400-11. Reveals the role of HDAC5 and MEF2C in SOST regulation.

76. Fei Y, Shimizu E, McBurney M, Partridge NC. Sirtuin 1 is a negative regulator of parathyroid hormone stimulation of matrix metalloproteinase 13 expression in osteoblastic cells. J Biol Chem. 2015;290(13):8373-82. Describes the function of a class III HDAC in PTH-stimulated MMP13 expression. 